\title{
Exact CS Reconstruction Condition of Undersampled Spectrum-Sparse Signals
}

\author{
Ying Luo,, Qun Zhang, ${ }^{1,2}$ Guozheng Wang, ${ }^{3}$ and Youqing Bai ${ }^{3}$ \\ ${ }^{1}$ Institute of Information and Navigation, Air Force Engineering University, Xian 710077, China \\ ${ }^{2}$ Key Laboratory for Information Science of Electromagnetic Waves, Ministry of Education, Fudan University, \\ Shanghai 200433, China \\ ${ }^{3}$ Institute of Science, Air Force Engineering University, Xian 710051, China
}

Correspondence should be addressed to Qun Zhang; zhangqunnus@gmail.com

Received 15 August 2013; Accepted 18 November 2013

Academic Editor: Feng Gao

Copyright (C) 2013 Ying Luo et al. This is an open access article distributed under the Creative Commons Attribution License, which permits unrestricted use, distribution, and reproduction in any medium, provided the original work is properly cited.

Compressive sensing (CS) reconstruction of a spectrum-sparse signal from undersampled data is, in fact, an ill-posed problem. In this paper, we mathematically prove that, in certain cases, the exact CS reconstruction of a spectrum-sparse signal from undersampled data is impossible. Then we present the exact CS reconstruction condition of undersampled spectrum-sparse signals, which is valuable for digital signal compression.

\section{Introduction}

In digital signal processing, the Nyquist sampling theorem indicates that the sampling rate must be twice as large as the bandwidth of the analog signal at least for acquiring the intact information of the signal. Restricted by the theorem, it is a challenge to digitize ultrawide bandwidth (UWB) signals because of the unfeasible high sampling rate requirement for the analog-to-digital converter (ADC). On the other hand, the mass sampling data have to be compressed to save the storage, which means that many data are abandoned in the compression processing. Hence, why not to obtain the compressed data of signals directly rather than to sample signal with ultrahigh rate and then abandon most of the samplings?

The emerging compressive sensing (CS) theory [1] provides an effective approach to solve this problem, which has attracted much attention recently [2-7]. Consider a signal $\mathbf{x} \in \mathbf{C}^{N \times 1}$ and assume it is sparse on an orthogonal basis $\Psi=\left\{\psi_{i}\right\}$ with $K$-sparse representation $(K \ll N)$ as $\mathbf{x}=$ $\boldsymbol{\Psi} \boldsymbol{\theta}$, where $\boldsymbol{\theta}$ is an $N \times 1$ column vector with $K$ nonzero elements. Let $\boldsymbol{\Phi}$ denote a measurement matrix and let $\mathbf{y}$ be the measurements vector of signal $\mathbf{x}$; it can be expressed as $\mathbf{y}=\boldsymbol{\Phi} \mathbf{x}=\boldsymbol{\Phi} \Psi \boldsymbol{\theta}$, where $\boldsymbol{\Phi}$ is a $M \times N$ matrix, $M$ denotes the number of measurements, and $K<M \ll N$. Therefore, the sampling rate is reduced significantly compared with Nyquist rate. Generally, recovery of the signal $\mathbf{x}$ from the measurements y is ill-posed because $M \ll N$ [8]. However, the CS theory demonstrates that if $\Phi \Psi$ has the Restricted Isometry Property (RIP), then it is indeed possible to recover the $K$ largest $\theta_{x}(i)$ when $M$ is large enough $[3,9]$. It is difficult to validate if a measurement matrix satisfies the RIP constraints given in [9] directly, but fortunately, the RIP is closely related to an incoherency between $\Phi$ and $\Psi$, where the rows of $\Phi$ do not provide a sparse representation of the columns of $\Psi$ and vice versa [4]. Furthermore, to ensure exact reconstruction, two different $K$-sparse signals may not be projected by a measurement matrix into the same sampling ensemble $[1,2,10]$.

When $\mathbf{x}$ is sparse in spectrum, the $N$-dimensional inverse discrete Fourier transform (IDFT) matrix $\left(\mathbf{D}_{N}^{-1}\right)$ can be chosen as the sparse representation matrix $(\Psi)$. In this case, an easy way to obtain the compressed data of the signal is to undersample the signal with lower sampling rate than 
the Nyquist rate [2]. Therefore the measurement matrix is in fact a partial unit matrix [11]. It is important to investigate the mathematical properties of compressive sensing reconstruction for this kind of undersampled spectrum-sparse signal. In the following, we mathematically prove that the exact CS reconstruction of a spectrum-sparse signal from undersampled data is impossible under certain conditions. In order to reconstruct a spectrum-sparse signal from undersampled data exactly, the corresponding exact CS reconstruction condition is presented, which is valuable for digital signal compression.

\section{Inexact CS Reconstruction Cases}

When a signal $\mathbf{x}$ is sparse in spectrum, the IDFT matrix $\mathbf{D}_{N}^{-1}$ and partial unit matrix can be chosen as the sparse representation matrix and the measurement matrix, respectively. By defining the downsampling rate $r$ to be the ratio between the Nyquist rate and the undersampling rate, an inexact CS reconstruction case can be depicted as the following theorem.

Theorem 1. Suppose $\mathbf{x} \in \mathbf{C}^{N \times 1}$ with Nyquist sampling rate $f_{s}$ is K-sparse in spectrum domain; $\mathbf{y}=\left\{x_{m r+b} \mid m r+b \leq\right.$ $N, m>0, m \in \mathbb{N}\}$ is an arbitrary subset of $\mathbf{x}$, where $r$ is the downsampling rate, $r \geq 2$, and $r \in \mathbb{N}, b$ is a constant and $b \in \mathbb{N}$, and $\mathbb{N}$ is the set of all natural numbers. $\mathbf{x}$ cannot be exactly reconstructed from $\mathbf{y}$ by CS.

Proof. According to the expression of $\mathbf{y}$, we have

$$
\mathbf{y}_{M \times 1}=\boldsymbol{\Phi}_{M \times N} \mathbf{x}_{N \times 1}=\Phi_{M \times N} \Psi_{N \times N} \boldsymbol{\theta}_{N \times 1}
$$

where $\boldsymbol{\theta}$ is $K$-sparse; $\boldsymbol{\Phi}$ is the measurement matrix with size $M \times N$ :

$$
\begin{aligned}
& \text { Column } 1 \quad r+1 \quad 2 r+1(M-1) r+1 \\
& \text { index }
\end{aligned}
$$

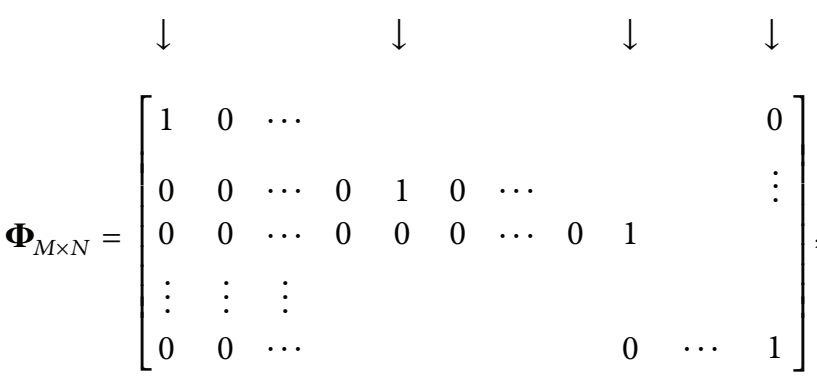

$$
\begin{aligned}
& \Psi_{N \times N}=D_{N}^{-1}=\frac{1}{N}\left[\begin{array}{ccccc}
1 & 1 & 1 & \cdots & 1 \\
1 & W_{N}^{-1} & W_{N}^{-2} & \cdots & W_{N}^{(N-1)} \\
1 & W_{N}^{-2} & W_{N}^{-4} & \ldots & W_{N}^{-2(N-1)} \\
\vdots & \vdots & \vdots & \vdots & \vdots \\
1 & W_{N}^{-(N-1)} & W_{N}^{-2(N-1)} & \ldots & W_{N}^{-(N-1)^{2}}
\end{array}\right] \text {, }
\end{aligned}
$$

Equation (1) is equivalent to

$$
\boldsymbol{\theta}_{M \times 1}^{\prime}=\mathbf{D}_{M} \mathbf{y}_{M \times 1}=\mathbf{D}_{M} \boldsymbol{\Phi}_{M \times N} \mathbf{D}_{N}^{-1} \boldsymbol{\theta}_{N \times 1},
$$

where $\mathbf{D}_{M}$ is the $M$-dimensional DFT matrix. Because $\mathbf{y}$ is isometrically downsampled from $\mathbf{x}, \boldsymbol{\theta}^{\prime}$ is also sparse. In (3), we define a new measurement matrix $\boldsymbol{\Phi}_{1}=\mathbf{D}_{M} \boldsymbol{\Phi}_{M \times N}$ and it can be expressed as

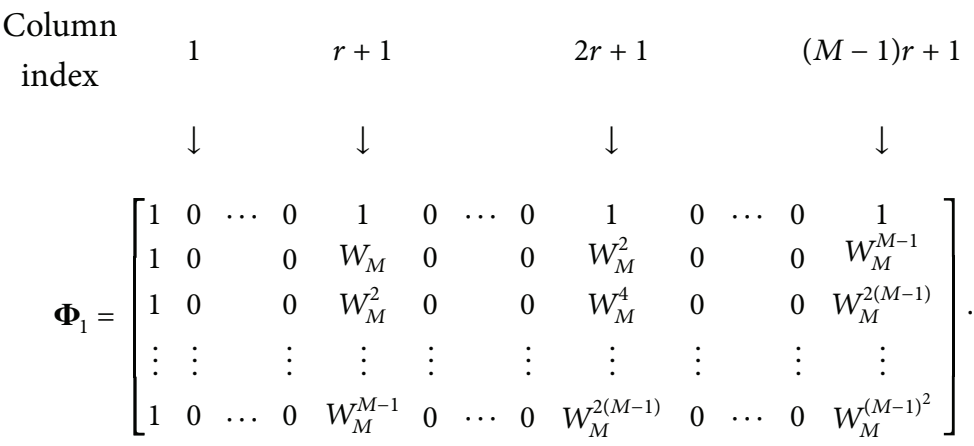

According to CS theory, if $\boldsymbol{\Phi}_{1} \mathbf{D}_{N}^{-1}$ satisfies the RIP, $\boldsymbol{\theta}$ can be exactly reconstructed. Let $\phi_{1 a}$ be the ath row of $\Phi_{1}$; we have

$$
\begin{aligned}
\boldsymbol{\phi}_{1 a}^{T} & =\mathbf{D}_{N}^{-1} \mathbf{D}_{N} \boldsymbol{\phi}_{1 a}^{T}=\mathbf{D}_{N}^{-1}\left(\mathbf{D}_{N} \boldsymbol{\phi}_{1 a}^{T}\right) \\
& =\mathbf{D}_{N}^{-1}\left[0, \frac{1-W_{N}^{M r}}{1-W_{M}^{a-1} W_{N}^{r}}, \ldots, \frac{1-W_{N}^{(N-1) M r}}{1-W_{M}^{a-1} W_{N}^{(N-1) r}}\right]^{T},
\end{aligned}
$$

where $W_{M}=\exp (-j 2 \pi / M)$. Because $\operatorname{Rank}\left(\mathbf{D}_{N}^{-1}\right)=N$, according to Cramer's Rule, the equation $\mathbf{D}_{N}^{-1} \boldsymbol{\alpha}=\boldsymbol{\phi}_{1 a}^{T}$ has unique solution; that is, 


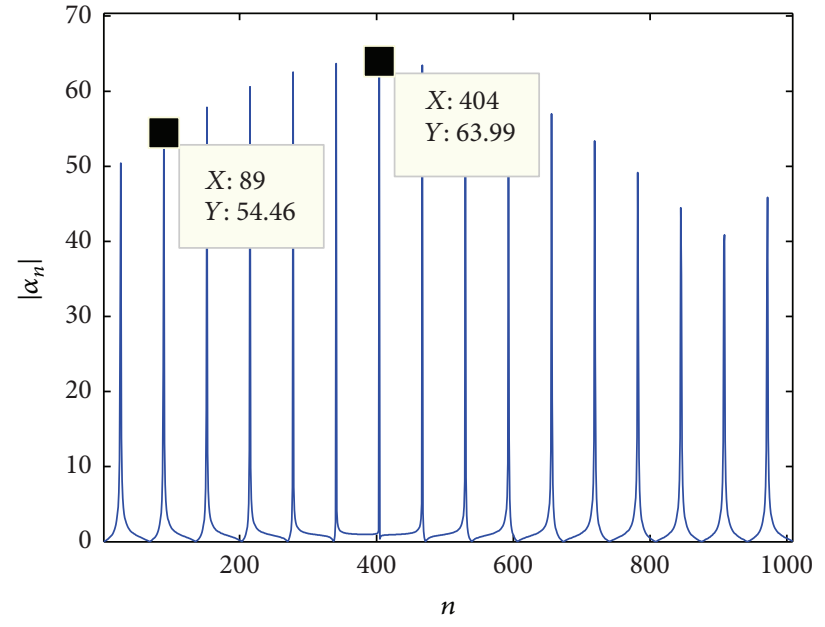

(a)

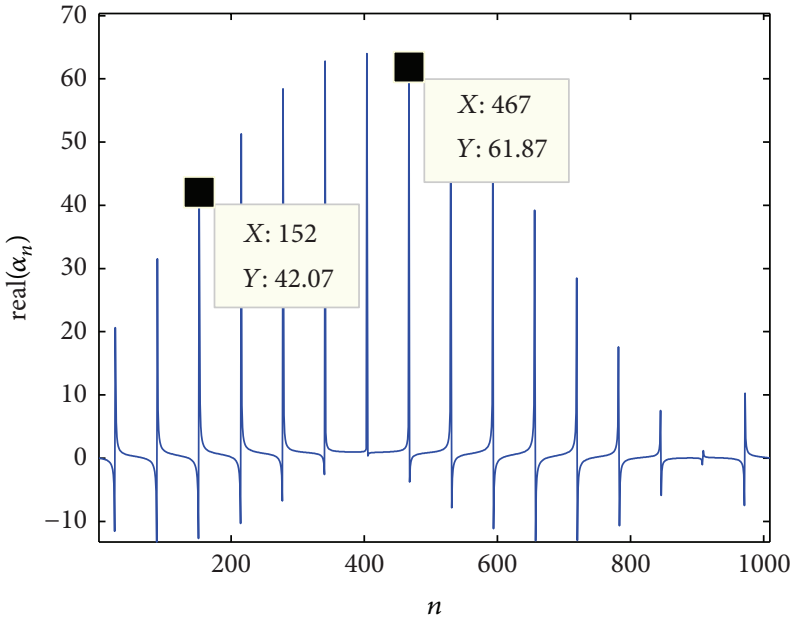

(b)

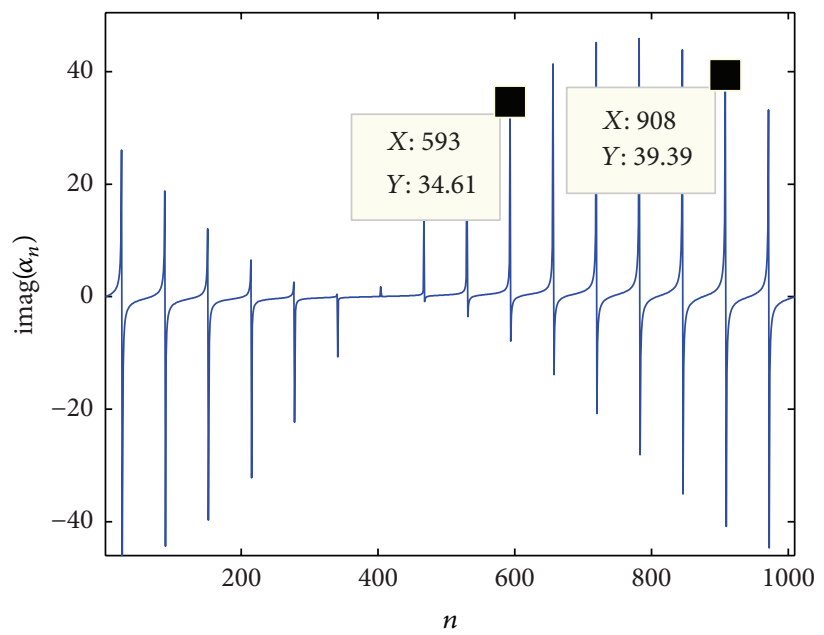

(c)

Figure 1: The sparsity of $\alpha_{n}$ when $N=1024, M=64, r=16$, and $a=40$. (a) $\left\{\left|\alpha_{n}\right|\right\} ;$ (b) $\left\{\operatorname{real}\left(\alpha_{n}\right)\right\} ;(\mathrm{c})\left\{\operatorname{imag}\left(\alpha_{n}\right)\right\}$.

Let $\alpha_{n}$ be the $n$th element of $\boldsymbol{\alpha}$; it can be obtained

$$
\begin{aligned}
\left|\alpha_{n}\right| & =\left|\frac{1-W_{N}^{(n-1) M r}}{1-W_{M}^{a-1} W_{N}^{(n-1) r}}\right| \\
& =\frac{1-\cos ((2 \pi / N)(n-1) M r)}{1-\cos (2 \pi((a-1) / M+((n-1) r) / N))} .
\end{aligned}
$$

It peaks at

$$
\frac{(n-1) r}{N}=-\frac{a-1}{M}+l, \quad l \in \mathbb{Z},
$$

where $\mathbb{Z}$ is set of all nonnegative integers. Because $N=(M-$ 1) $r+1$, we have

$$
n=-a+2+\frac{(r-1)(a-1)}{M r}+l\left(M-1+\frac{1}{r}\right)
$$

and $n$ is a natural number; hence

$$
n=\left[-a+2+\frac{(r-1)(a-1)}{M r}+l\left(M-1+\frac{1}{r}\right)\right],
$$

where $[(\cdot)]$ returns the round of $(\cdot)$. When $n$ does not satisfy (10), $\left|\alpha_{n}\right|$ is relatively quite small. It indicates that $\left\{\left|\alpha_{n}\right|\right\}$ is sparse. Similarly, it can be proved that both $\left\{\operatorname{real}\left(\alpha_{n}\right)\right\}$ and $\left\{\operatorname{imag}\left(\alpha_{n}\right)\right\}$ are also sparse. Therefore, $\phi_{1 a}$ can be sparsely represented by the columns of $\mathbf{D}_{N}^{-1}$; that is, $\boldsymbol{\Phi}_{1} \mathbf{D}_{N}^{-1}$ does not satisfy the RIP, and $\boldsymbol{\theta}$ cannot be exactly reconstructed.

Figure 1 shows the values of $\left\{\left|\alpha_{n}\right|\right\},\left\{\operatorname{real}\left(\alpha_{n}\right)\right\}$, and $\left\{\operatorname{imag}\left(\alpha_{n}\right)\right\}$ when $N=1024, M=64, r=16$, and $a=40$. It is obvious that $\left\{\left|\alpha_{n}\right|\right\},\left\{\operatorname{real}\left(\alpha_{n}\right)\right\}$, and $\left\{\operatorname{imag}\left(\alpha_{n}\right)\right\}$ are sparse and the locations of peaks agree well with the theoretical values determined by (10).

By Theorem 1 and its proof, we can also obtain the following corollary.

Corollary 2. Suppose $\mathbf{x} \in \mathbf{C}^{N \times 1}$ with Nyquist sampling rate $f_{s}$ is K-sparse in spectrum domain; $\mathbf{x}^{\prime}=\left\{x_{m r+b} \mid m r+b \leq\right.$ $N, m>0, m \in \mathbb{N}\}$ is an arbitrary subset of $\mathbf{x}$, where $r$ is the downsampling rate, $r \geq 2$, and $r \in \mathbb{N}$ and $b$ is a constant and 
$b \in \mathbb{N}$. Let $\mathbf{y}$ be an arbitrary subset of $\mathbf{x}^{\prime}$; then $\mathbf{x}$ cannot be exactly reconstructed from $\mathbf{y}$ by $C S$.

Proof. Assume the length of $\mathbf{x}^{\prime}$ is $M$ and we have

$$
\mathbf{y}=\Phi_{2} \mathbf{x}^{\prime}=\Phi_{2} \Phi D_{N}^{-1} \boldsymbol{\theta}
$$

where $\Phi_{2}$ is a partial unit matrix. Assuming the size of $\Phi_{2}$ is $L \times M(L \leq M)$, the solution set of (1) is a subset of the solution set of (11). According to Theorem 1, the solutions of (1) are not determined; therefore, $\boldsymbol{\theta}$ in (11) cannot be determined and $\mathbf{x}$ cannot be exactly reconstructed.

Corollary 2 indicates that, if the set of undersamplings is only a subset of the set of signals' samplings with sampling rate lower than the Nyquist rate, the signal cannot be exactly reconstructed from these undersamplings by CS. Therefore, when designing the ADC with random sampling space, the ADC should better possess the capability with the minimum sampling space of $1 / f_{s}$, where $f_{s}$ is the Nyquist rate. Taken in this sense, the high sampling rate requirement for ADC is indeed not suppressed even though the CS theory is utilized.

\section{Exact CS Reconstruction Condition}

In the following, we present the exact CS reconstruction condition of undersampled spectrum-sparse signals.

Theorem 3. Suppose $\mathbf{x} \in \mathbf{C}^{N \times 1}$ with Nyquist sampling rate $f_{s}$ is $K$-sparse in spectrum domain; and the frequency indexes of $K$ nonzero points in spectrum are $f_{h}(h=1,2, \ldots, K)$. Undersampling $\mathbf{x}$ with rate $f_{s} / r_{1}, f_{s} / r_{2}, \ldots, f_{s} / r_{i}, \ldots, f_{s} / r_{I}$ $\left(i=1,2, \ldots, I, r_{i} \geq 2\right.$, and $\left.r_{i} \in \mathbb{N}\right)$, respectively, all the samples consist of $\mathbf{y}_{M \times 1}$. The necessary and sufficient condition for $\mathbf{x}$ exactly reconstructed from $\mathbf{y}$ by $C S$ is

$$
\begin{aligned}
& \left\{f_{h}+\frac{k_{1} f_{s}}{r_{1}}\left|h=1,2, \ldots, K ; k_{1} \in \mathbb{Z} ;\right| f_{h}+\frac{k_{1} f_{s}}{r_{1}} \mid \leq \frac{f_{s}}{2}\right\} \\
& \cap\left\{f_{h}+\frac{k_{2} f_{s}}{r_{2}}\left|h=1,2, \ldots, K ; k_{2} \in \mathbb{Z} ;\right| f_{h}+\frac{k_{2} f_{s}}{r_{2}} \mid \leq \frac{f_{s}}{2}\right\} \\
& \cap \ldots \\
& \cap\left\{f_{h}+\frac{k_{I} f_{s}}{r_{I}}\left|h=1,2, \ldots, K ; k_{I} \in \mathbb{Z} ;\right| f_{h}+\frac{k_{I} f_{s}}{r_{I}} \mid \leq \frac{f_{s}}{2}\right\} \\
& =\left\{f_{h} \mid h=1,2, \ldots, K\right\} .
\end{aligned}
$$

Proof. Reconstructing $\mathbf{x}$ from $\mathbf{y}$ is in fact to solve the following underdetermined equation system:

$$
\begin{array}{r}
\mathbf{y}_{1}=\boldsymbol{\Phi}_{1} \mathbf{x}=\boldsymbol{\Phi}_{1} \mathbf{D}_{N}^{-1} \boldsymbol{\theta} \\
\mathbf{y}_{2}=\Phi_{2} \mathbf{x}=\Phi_{2} \mathbf{D}_{N}^{-1} \boldsymbol{\theta} \\
\vdots \\
\mathbf{y}_{I}=\Phi_{I} \mathbf{x}=\Phi_{I} D_{N}^{-1} \boldsymbol{\theta}
\end{array}
$$

where $\boldsymbol{\Phi}_{i}$ is the measurement matrix according to the undersampling rate $f_{s} / r_{i} ; \mathbf{y}_{i}$ is composed of the undersamplings of $\mathbf{x}$ with rate $f_{s} / r_{i}$. Assume the frequency indexes of the solution set of the $i$ th equation are $\left\{\widehat{f}_{h i}\right\}$; because $\mathbf{y}_{i}$ is the isometric downsampling from $\mathbf{x}$, we have

$$
\begin{aligned}
& \left\{\widehat{f}_{h i}\right\} \\
& \quad \subseteq\left\{f_{h}+\frac{k_{i} f_{s}}{r_{i}}\left|h=1,2, \ldots, K ; k_{i} \in \mathbb{Z} ;\right| f_{h}+\frac{k_{i} f_{s}}{r_{i}} \mid \leq \frac{f_{s}}{2}\right\} .
\end{aligned}
$$

Hence, the solution set of (13) is

$$
\begin{gathered}
\cap\left\{\widehat{f}_{h i}\right\} \subseteq \cap\left\{f_{h}+\frac{k_{i} f_{s}}{r_{i}} \mid h=1,2, \cdots, K ; k_{i} \in \mathbb{Z} ;\right. \\
\left.\left|f_{h}+\frac{k_{i} f_{s}}{r_{i}}\right| \leq \frac{f_{s}}{2}\right\} .
\end{gathered}
$$

Only if (12) holds true, under the constraint of $K$-sparse, it yields $\cap\left\{\widehat{f}_{h i}\right\}=\left\{f_{h} \mid h=1,2, \ldots, K\right\}$; therefore, $\mathbf{x}$ is exactly reconstructed.

Theorem 3 indicates that it is possible to reconstruct a spectrum-sparse signal exactly from its multirate downsamplings. It means that, when condition (12) is satisfied, the multirate downsampling can be used to compress the data of digital signals. The conclusion is valuable to the design of the ADC when the analog signal is a priori known sparse in spectrum.

In order to validate Theorem 3, an experiment is given as follows. In the experiment, the signal $x(t)$ with Nyquist sampling rate $f_{s}=1 \mathrm{~Hz}$ is expressed as $x(t)=\exp (0.2 \times$ $2 \pi t)+0.8 \exp (0.45 \times 2 \pi t)+0.6 \exp (0.3 \times 2 \pi t), t \in[0,1023]$. The sparsity of $x(t)$ in spectrum domain is 3 and $f_{h}=\{0.2$, $0.45,0.3\}$. The spectrum of $x(t)$ is shown in Figure $2(\mathrm{a})$. Let $r_{1}=7$ and $r_{2}=10$. Therefore, we have

$$
\begin{aligned}
\left\{f_{h}+\right. & \left.\frac{k_{1} f_{s}}{r_{1}}\left|h=1,2, \cdots, K ; k_{1} \in \mathbb{Z} ;\right| f_{h}+\frac{k_{1} f_{s}}{r_{1}} \mid \leq \frac{f_{s}}{2}\right\} \\
= & \left\{0.2+\frac{k_{1}}{7}\left|k_{1} \in \mathbb{Z} ;\right| 0.2+\frac{k_{1}}{7} \mid \leq \frac{1}{2}\right\} \\
\cap & \left\{0.45+\frac{k_{1}}{7}\left|k_{1} \in \mathbb{Z} ;\right| 0.45+\frac{k_{1}}{7} \mid \leq \frac{1}{2}\right\} \\
\cap & \left\{0.3+\frac{k_{1}}{7}\left|k_{1} \in \mathbb{Z} ;\right| 0.3+\frac{k_{1}}{7} \mid \leq \frac{1}{2}\right\} \\
= & \left\{0.2,0.2+\frac{1}{7}, 0.2+\frac{2}{7}, 0.2-\frac{1}{7}, 0.2-\frac{2}{7},\right. \\
& 0.2-\frac{3}{7}, 0.2-\frac{4}{7}, 0.45,0.45-\frac{1}{7}, \\
& 0.45-\frac{2}{7}, 0.45-\frac{3}{7}, 0.45-\frac{4}{7}, 0.45-\frac{5}{7}, \\
& 0.45-\frac{6}{7}, 0.3,0.3+\frac{1}{7}, 0.3-\frac{1}{7}, 0.3-\frac{2}{7}, \\
& \left.0.3-\frac{3}{7}, 0.3-\frac{4}{7}, 0.3-\frac{5}{7}\right\},
\end{aligned}
$$




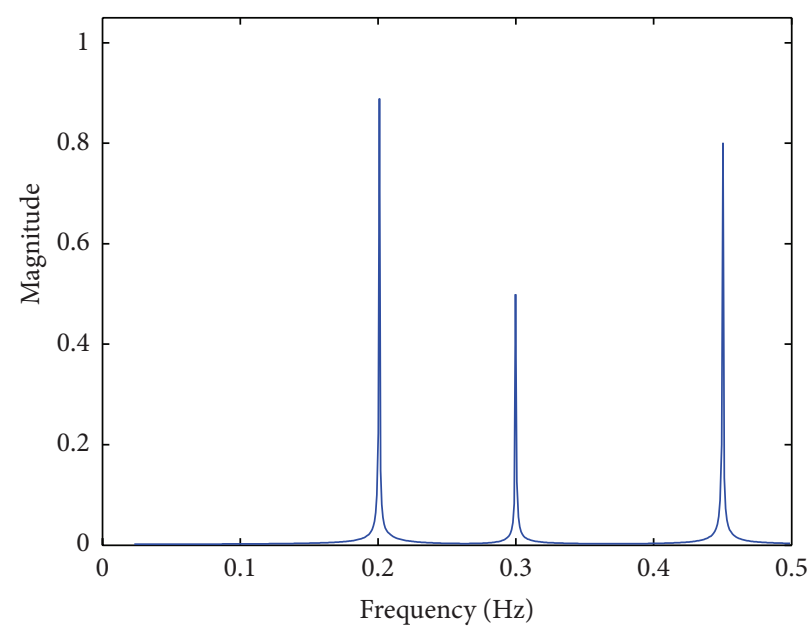

(a)

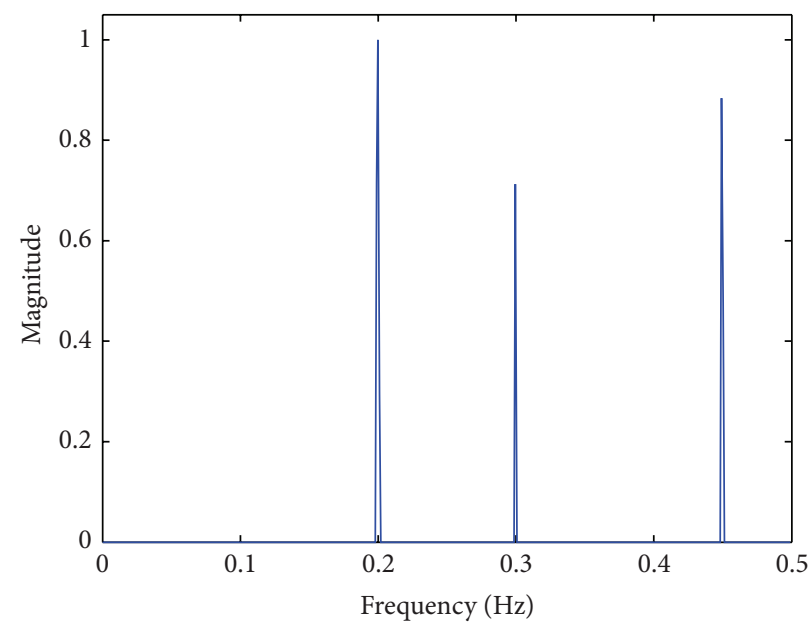

(b)

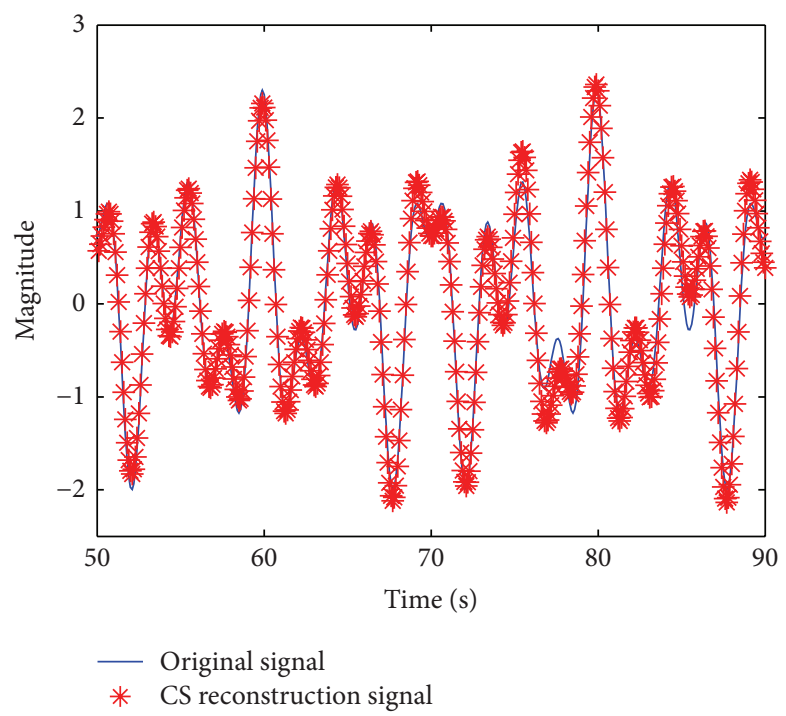

(c)

Figure 2: Experiment for validation of Theorem 3. (a) The spectrum of the signal $x(t)$ with Nyquist sampling rate; (b) the spectrum of the reconstructed signal by CS; (c) the comparison of $x(t)$ and the reconstructed signal in time domain.

$$
\begin{aligned}
\left\{f_{h}+\right. & \left.\frac{k_{2} f_{s}}{r_{2}}\left|h=1,2, \ldots, K ; k_{2} \in \mathbb{Z} ;\right| f_{h}+\frac{k_{2} f_{s}}{r_{2}} \mid \leq \frac{f_{s}}{2}\right\} \\
= & \left\{0.2+\frac{k_{2}}{10}\left|k_{2} \in \mathbb{Z} ;\right| 0.2+\frac{k_{2}}{10} \mid \leq \frac{1}{2}\right\} \\
\cap & \left\{0.45+\frac{k_{2}}{10}\left|k_{2} \in \mathbb{Z} ;\right| 0.45+\frac{k_{2}}{10} \mid \leq \frac{1}{2}\right\} \\
\cap & \left\{0.3+\frac{k_{2}}{10}\left|k_{2} \in \mathbb{Z} ;\right| 0.3+\frac{k_{2}}{10} \mid \leq \frac{1}{2}\right\} \\
= & \{-0.5,-0.4,-0.3,-0.2,-0.1,0,0.1,0.2,0.3, \\
& \quad 0.4,0.5,0.45,0.35,0.25,0.15,0.05,-0.05, \\
& -0.15,-0.25,-0.35,-0.45\} .
\end{aligned}
$$

Obviously, the intersection of (16) is $f_{h}=\{0.2,0.45,0.3\}$. Hence, $x(t)$ can be exactly reconstructed from the downsamplings. In the experiment, we choose the orthogonal matching pursuit (OMP) algorithm [12] to reconstruct $x(t)$ from the downsamplings. The spectrum of the reconstructed signal by CS is shown in Figure 2(b), which is very close to that in Figure 2(a). The comparison of the original signal $x(t)$ with Nyquist sampling rate and the reconstructed signal in time domain is also given in Figure 2(c). From the figure, it can be found that the reconstructed signal is close to the original signal, which validates the correctness of Theorem 3.

\section{Conflict of Interests}

The authors declare that there is no conflict of interests regarding the publication of this paper. 


\section{Acknowledgments}

This research was supported in part by the National Natural Science Foundation of China under Grant 61201369 and 61172169 and in part by the Natural Science Foundation Research Program of Shaanxi Province under Grant 2013JQ8008.

\section{References}

[1] D. L. Donoho, "Compressed sensing," IEEE Transactions on Information Theory, vol. 52, no. 4, pp. 1289-1306, 2006.

[2] E. J. Candès, J. Romberg, and T. Tao, "Robust uncertainty principles: exact signal reconstruction from highly incomplete frequency information," IEEE Transactions on Information Theory, vol. 52, no. 2, pp. 489-509, 2006.

[3] E. J. Candès and T. Tao, "Near-optimal signal recovery from random projections: universal encoding strategies?" IEEE Transactions on Information Theory, vol. 52, no. 12, pp. 5406-5425, 2006.

[4] R. Baraniuk and P. Steeghs, "Compressive radar imaging," in IEEE Radar Conference, pp. 128-133, Boston, Mass, USA, 2007.

[5] M. A. T. Figueiredo, R. D. Nowak, and S. J. Wright, "Gradient projection for sparse reconstruction: application to compressed sensing and other inverse problems," IEEE Journal of Selected Topics in Signal Processing, vol. 1, no. 4, pp. 586-597, 2007.

[6] Y. Zhang, "Theory of compressive sensing via $\mathscr{L} 1$-minimization: a non-RIP analysis and extensions," Journal of the Operations Research Society of China, vol. 1, no. 1, pp. 79-105, 2013.

[7] T. Strohmer, "Measure what should be measured: progress and challenges in compressive sensing," IEEE Signal Processing Letters, vol. 19, no. 12, pp. 887-893, 2012.

[8] B. Adcock, A. C. Hansen, E. Herrholz, and G. Teschke, "Generalized sampling: extension to frames and inverse and ill-posed problems," Inverse Problems, vol. 29, no. 1, Article ID 015008, p. 27, 2013.

[9] E. J. Candès and T. Tao, "Decoding by linear programming," IEEE Transactions on Information Theory, vol. 51, no. 12, pp. 4203-4215, 2005.

[10] R. Baraniuk, M. Davenport, R. DeVore, and M. Wakin, "A simple proof of the restricted isometry property for random matrices," Constructive Approximation, vol. 28, no. 3, pp. 253263, 2008.

[11] Y. Luo, Q. Zhang, W. Hong, and Y.-R. Wu, "Waveform design and high-resolution imaging of cognitive radar based on compressive sensing," Science China Information Sciences, vol. 55, no. 11, pp. 2590-2603, 2012.

[12] J. A. Tropp and A. C. Gilbert, "Signal recovery from random measurements via orthogonal matching pursuit," IEEE Transactions on Information Theory, vol. 53, no. 12, pp. 4655-4666, 2007. 


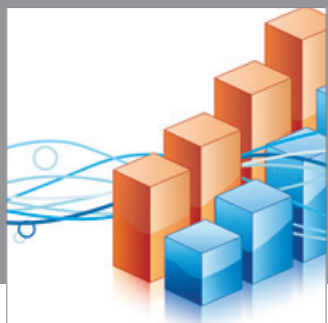

Advances in

Operations Research

mansans

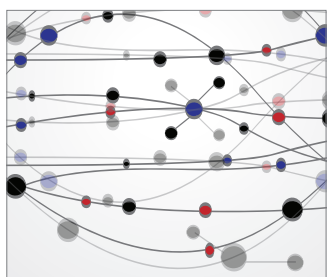

The Scientific World Journal
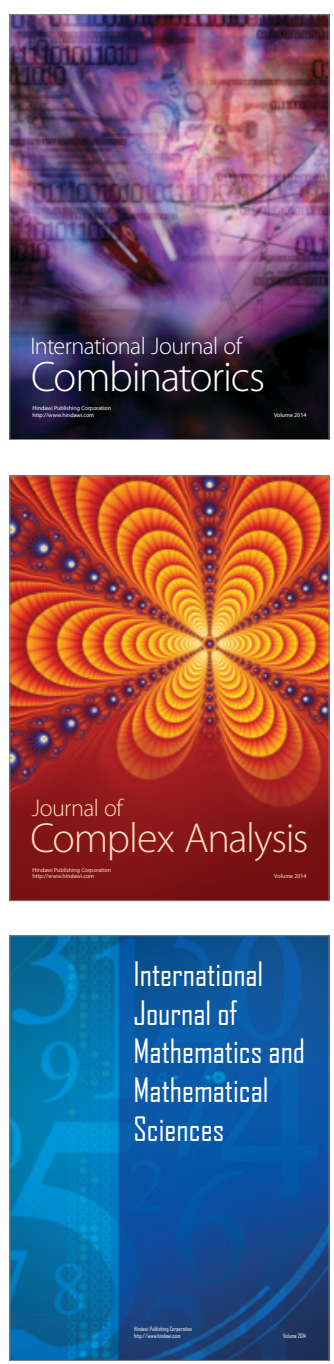
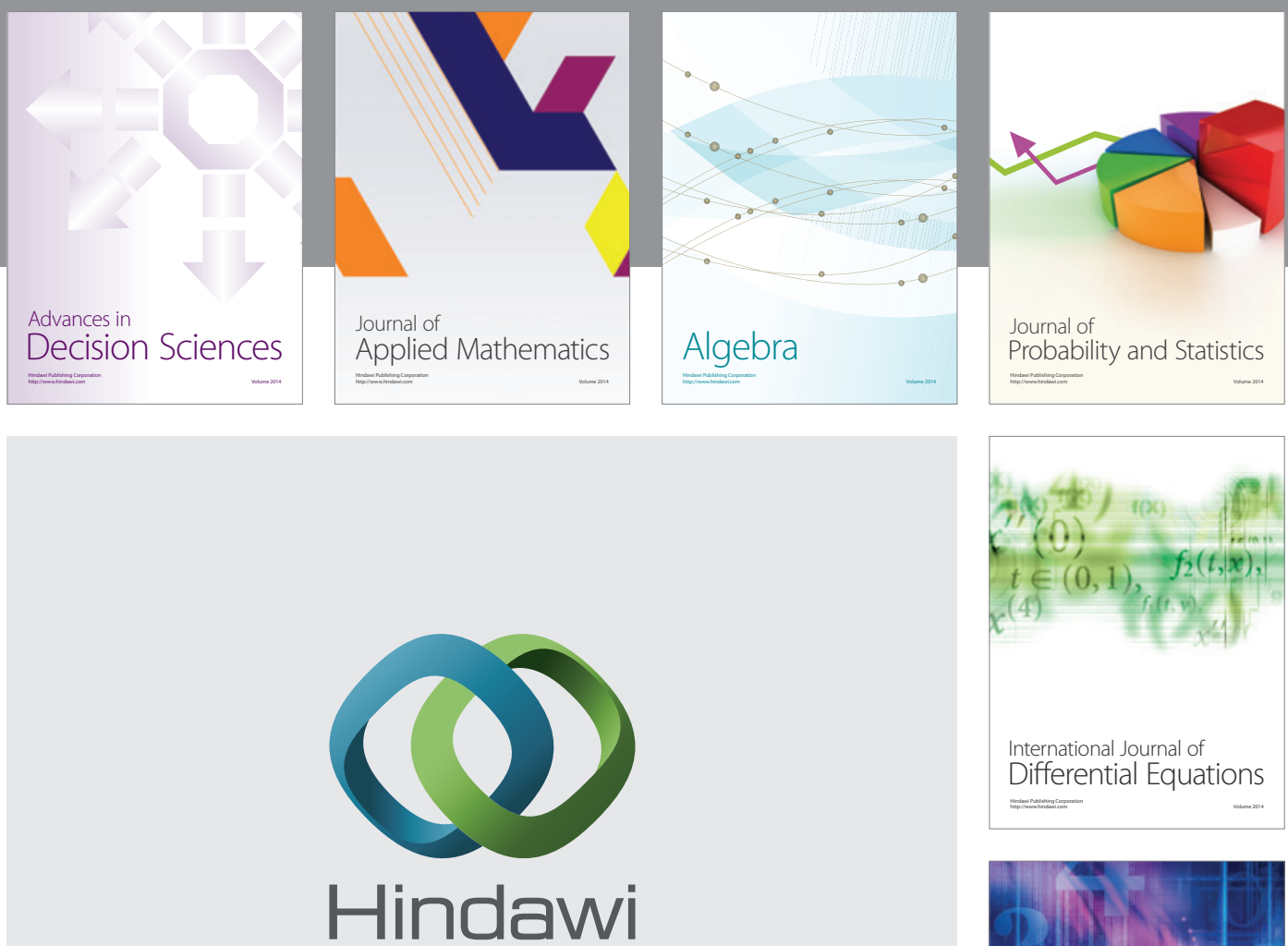

Submit your manuscripts at http://www.hindawi.com
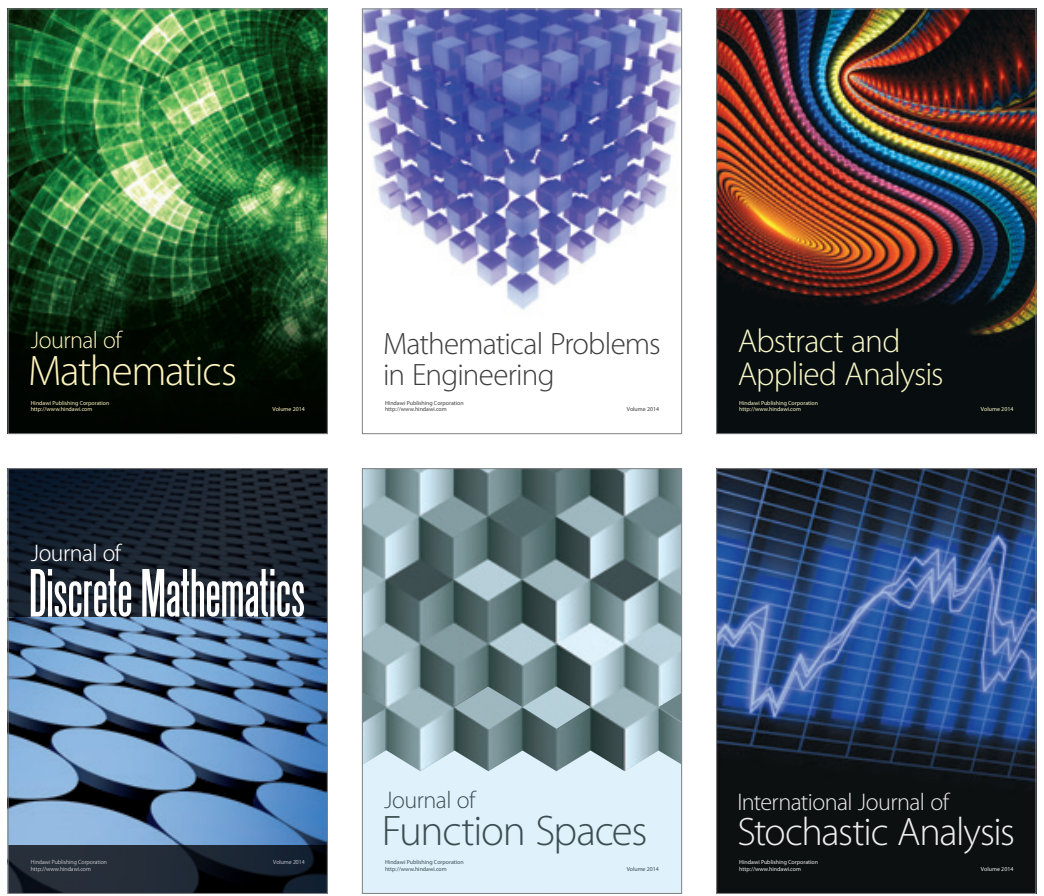

Journal of

Function Spaces

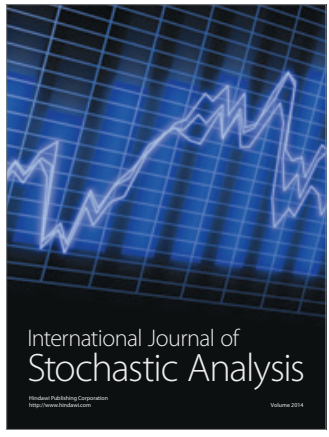

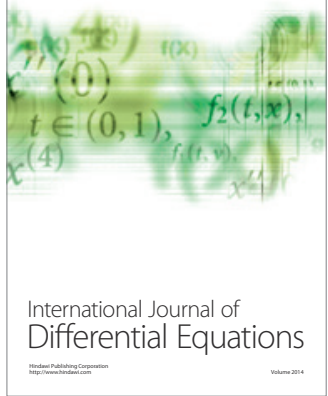
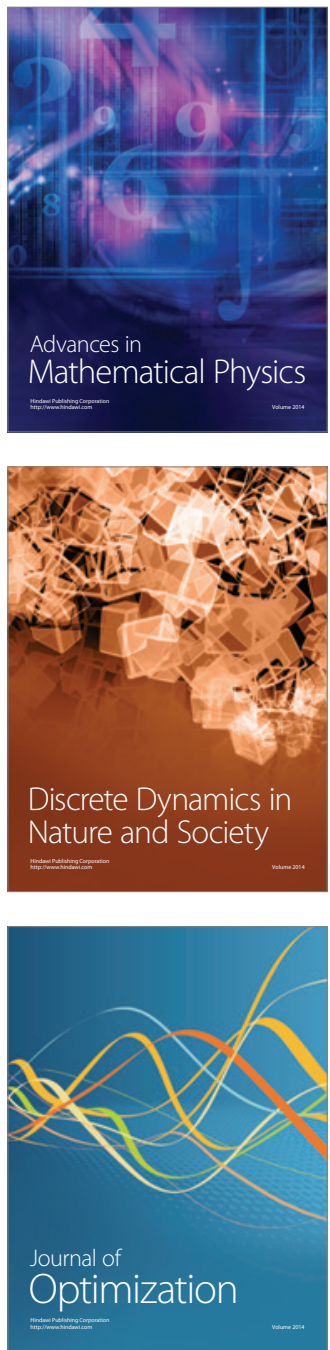